PROCEEDINGS OF THE

AMERICAN MATHEMATICAL SOCIETY

Volume 135, Number 11, November 2007, Pages 3711-3721

S $0002-9939(07) 08865-\mathrm{X}$

Article electronically published on June 21, 2007

\title{
ON THE COMPUTABILITY-THEORETIC COMPLEXITY OF TRIVIAL, STRONGLY MINIMAL MODELS
}

\author{
BAKHADYR M. KHOUSSAINOV, MICHAEL C. LASKOWSKI, STEFFEN LEMPP, \\ AND REED SOLOMON
}

(Communicated by Julia Knight)

\begin{abstract}
We show the existence of a trivial, strongly minimal (and thus uncountably categorical) theory for which the prime model is computable and each of the other countable models computes $\mathbf{0}^{\prime \prime}$. This result shows that the result of Goncharov/Harizanov/Laskowski/Lempp/McCoy (2003) is best possible for trivial strongly minimal theories in terms of computable model theory. We conclude with some remarks about axiomatizability.
\end{abstract}

Both vector spaces and algebraically closed fields have the property that the uncountable structures in these classes are determined up to isomorphism by their cardinality. Model theory provides a general framework in which to study this behavior. A first order theory $T$ is called $\kappa$-categorical (for an infinite cardinal $\kappa$ ) if $\mathcal{A} \cong \mathcal{B}$ whenever $\mathcal{A}, \mathcal{B} \models T$ and $|\mathcal{A}|=|\mathcal{B}|=\kappa$. Morley Mo65. proved that if $T$ is categorical in some uncountable cardinality, then it is categorical in all uncountable cardinalities. (We assume here and for the rest of this paper that the language is countable.) Therefore, any $\omega_{1}$-categorical theory has a unique model of each uncountable cardinality.

Baldwin and Lachlan BL71] showed that for an uncountably categorical theory $T$, either there is a unique countable model of $T$, or the countable models of $T$ form an elementary chain of length $\omega+1: \mathcal{A}_{0} \prec \mathcal{A}_{1} \prec \cdots \prec \mathcal{A}_{\omega}$, where $\mathcal{A}_{0}$ is the prime (or atomic) model and $\mathcal{A}_{\omega}$ is the countably saturated model. The examples of vector spaces (over a fixed countable field) and algebraically closed fields fall into this second category, with the models in the elementary chain determined by the cardinality of a basis and by the transcendence degree. It is this case of uncountably categorical but not $\omega$-categorical theories that we are interested in.

Together, Morley's Categoricity Theorem and the Lachlan-Baldwin Theorem give a good algebraic picture of the infinite models of an uncountably categorical theory $T$. However, there are additional questions to consider about the complexity

Received by the editors December 14, 2005 and, in revised form, January 19, 2006 and August 4, 2006.

2000 Mathematics Subject Classification. Primary 03C57; Secondary 03D45.

Key words and phrases. Computable model, uncountably categorical, strongly minimal, trivial geometry, axiomatizability.

The first author's research was partially supported by The Marsden Fund of New Zealand.

The second author's research was partially supported by NSF grant DMS-0300080.

The third author's research was partially supported by NSF grant DMS-0140120.

The fourth author's research was partially supported by NSF grant DMS-0400754. 
of such theories and models. For example, from a model-theoretic point of view, one can ask about the complexity of sentences required to axiomatize these theories, and from a computability-theoretic point of view, one can ask about the complexity of constructing the models of such a theory.

To address the latter question, we recall that a countable model is said to be decidable if it has an isomorphic copy with universe $\omega$ for which the elementary diagram forms a computable set of formulas; and computable if the open diagram of some such copy forms a computable set of formulas. (We assume that we are working in a computable language, i.e., that the arity of each relation and function symbol in the language is uniformly computable. Note that these notions easily relativize to the notions of "d-decidable" and "d-computable" for any Turing degree $\mathbf{d}$.)

Harrington Ha74 and Khisamiev Kh74] showed that all countable models of a decidable uncountably categorical theory are decidable. It follows trivially from the definitions that if an uncountably categorical theory has a decidable model, then the theory is decidable, and so all its countable models are decidable.

In contrast, Goncharov Go78 first exhibited an uncountably categorical theory for which one but not all countable models are computable; for his theory, the prime model is computable while all other countable models are only $\mathbf{0}^{\prime}$-computable. More examples of uncountably categorical theories for which some but not all countable models are computable were found by Kudaibergenov Ku80, Khoussainov/Nies/Shore KNS97, Nies [Ni99 and others. These theories have three properties in common: They use infinite languages, they are trivial and strongly minimal (defined below), and all the countable models are computable from $\mathbf{0}^{\prime}$.

Herwig/Lempp/Ziegler [HLZ99] showed that the use of an infinite language is not essential by constructing a theory in a language of three binary relation symbols for which the prime model is computable but all nonprime models are only $\mathbf{0}^{\prime}$ computable. Moreover, they showed that for any Turing degree $\mathbf{a} \leq \mathbf{0}^{\prime}$, there is a trivial strongly minimal theory $T$ in this language such that the prime model of $T$ is computable while (the open diagrams of) all the nonprime models have copies in exactly the degrees $\geq \mathbf{a}$, i.e., the nonprime models all "code" the degree $\mathbf{a}$.

The second common property of these theories is that each example is trivial and strongly minimal. A theory $T$ is called strongly minimal if every definable subset (with parameters) of each model $\mathcal{A}=T$ is either finite or cofinite. Such a model $\mathcal{A} \models T$ is trivial (or has trivial pregeometry) if the algebraic closure of any subset $D \subseteq A$, denoted by $\operatorname{acl}(D)$, is the union of the algebraic closures of the elements of $D$. (This property depends only on the theory of $\mathcal{A}$. An element $a \in A$ is algebraic over $D$ if there are parameters $\bar{d} \in D$ and a formula $\psi(x, \bar{d})$ such that $\mathcal{A} \models \psi(a, \bar{d})$ and the set of all $b \in A$ for which $\mathcal{A}=\psi(b, \bar{d})$ is finite. The algebraic closure of $D$ is the set of all elements which are algebraic over $D$. In the case of algebraically closed fields and vector spaces, $\operatorname{acl}(D)$ is the algebraic closure of the subfield generated by $D$, and the span of $D$, respectively. Buechler [Bu96] is a good reference on strongly minimal theories.) Strong minimality implies uncountable categoricity, so this property shows that it is possible to get the behaviors described above within a restricted class of uncountably categorical theories.

The third property is our main concern in this paper. Although all of the models in the examples above are $\mathbf{0}^{\prime}$-computable, it was not clear for several years why there should be any fixed arithmetic bound on the complexity of these models. 
The existence of such an upper bound was given by a purely model-theoretic result by Goncharov/Harizanov/Laskowski/Lempp/McCoy GHLLM03: If $T$ is a trivial strongly minimal theory, $\mathcal{M} \models T$ and $\operatorname{Th}\left(\mathcal{M}_{M}\right)$ denotes the theory of $\mathcal{M}$ in the expanded language which includes a constant symbol for each element of $\mathcal{M}$, then $\operatorname{Th}\left(\mathcal{M}_{M}\right)$ is model complete, and hence $\forall \exists$-axiomatizable (see, e.g., Chang/Keisler CK90, pp. 186-187]). Therefore, if $\mathcal{M} \models T$ is computable, then both $\operatorname{Th}\left(\mathcal{M}_{M}\right)$ and $\operatorname{Th}(\mathcal{M})=T$ are $\mathbf{0}^{\prime \prime}$-decidable. By the relativized version of the theorem of Harrington [Ha74] and Khisamiev Kh74, all countable models of $T$ are $\mathbf{0}^{\prime \prime}$-decidable. (This statement is stronger than saying they are $\mathbf{0}^{\prime \prime}$-computable; it says that each model has an isomorphic copy in which the elementary diagram (and not just the open diagram) is $\mathbf{0}^{\prime \prime}$-computable.)

There is a computability-theoretic gap between the results of Goncharov Go78] (in which the countable nonprime models of $T$ are computable in $\mathbf{0}^{\prime}$ ) and of Goncharov/Harizanov/Laskowski/Lempp/McCoy GHLLM03 (in which the upper bound of $\mathbf{0}^{\prime \prime}$ is given). Is it possible for a trivial strongly minimal theory to have a computable model and another countable model which is only $\mathbf{0}^{\prime \prime}$-computable? In this paper, we give an affirmative answer and thereby show that the result of Goncharov/Harizanov/Laskowski/Lempp/McCoy GHLLM03 is best possible in terms of computable model theory (i.e., the complexity of the countable models).

Theorem 1. There is a trivial, strongly minimal (and thus uncountably categorical) first-order theory $T$ such that the prime model of $T$ is computable and $\mathbf{0}^{\prime \prime}$ is computable from the atomic diagram of any nonprime countable model of $T$.

We prove this theorem in Section 1. As a corollary to this theorem, we can also conclude that the $\forall \exists$-axiomatization of the theory of a strongly minimal model in the expanded language naming constants is optimal, i.e., there is no axiomatization by Boolean combinations of $\forall$-formulas. However, this can already be seen from much simpler examples, e.g., the theory of an equivalence relation with all equivalence classes of size two. Section 2 contains some general remarks about axiomatizability by $\forall \exists$-formulas, $\forall$-formulas, and Boolean combinations of $\forall$-formulas.

\section{The proof of Theorem 1}

We begin by defining the type of family of sets which we will code into our theory.

Definition 2. An infinite family $\mathcal{F}$ of finite subsets of $\omega$ is an almost everywhere (or a.e.) family if for every $n \in \omega$, either $n \in X$ for all but finitely many $X \in \mathcal{F}$ or $n \notin X$ for all but finitely many $X \in \mathcal{F}$. Such a family $\mathcal{F}$ has an infinite limit if the set

$$
\begin{aligned}
I_{\mathcal{F}} & =\{n \in \omega \mid n \in X \text { for all but finitely many } X \in \mathcal{F}\} \\
& =\{n \in \omega \mid n \in X \text { for infinitely many } X \in \mathcal{F}\}
\end{aligned}
$$

is infinite.

To code this type of family, we generalize the notion of "cubes" introduced by Khoussainov/Nies/Shore KNS97. Fix a language $\mathcal{L}$ consisting of binary relation symbols $F_{i}$ (for $i \in \omega$ ), which we will all assume to be symmetric and irreflexive and to have in-degree and out-degree $\leq 1$, so we can think of them as coding permutations $f_{i}$ of order 2 on subsets of the model. (If we used function symbols $f_{i}$ in our language rather than relation symbols, then by the comments in Section 2 , 
the theory $\operatorname{Th}\left(\mathcal{M}_{M}\right)$ for a model $\mathcal{M} \models T_{\mathcal{F}}$ (defined below) would have a quantifierdepth 1 axiomatization and hence the most we can code into such a model is $\mathbf{0}^{\prime}$.)

Before giving the formal definition of an $S$-cube (for $S \subseteq \omega$ ), we give several concrete examples. Suppose $S=\left\{n_{0}\right\}$. In this case, an $S$-cube consists of two elements $a_{0}$ and $b_{0}$ such that $F_{n_{0}}\left(a_{0}, b_{0}\right)$ (and hence $F_{n_{0}}\left(b_{0}, a_{0}\right)$ by symmetry) and $\neg F_{m}\left(a_{0}, b_{0}\right)$ for all $m \neq n_{0}$ (and hence $\neg F_{m}\left(b_{0}, a_{0}\right)$ by symmetry). Notice that the function $f_{n_{0}}$ induced by $F_{n_{0}}$ and defined by $f_{n_{0}}\left(a_{0}\right)=b_{0}$ and $f_{n_{0}}\left(b_{0}\right)=a_{0}$ gives an $\mathcal{L}$-automorphism of the $S$-cube.

Next, suppose $S=\left\{n_{0}, n_{1}\right\}$. To construct the $S$-cube, we begin with two $\left\{n_{0}\right\}$ cubes consisting of the elements $a_{0}$ and $b_{0}$ connected by $F_{n_{0}}\left(a_{0}, b_{0}\right)$ and the elements $a_{1}$ and $b_{1}$ connected by $F_{n_{0}}\left(a_{1}, b_{1}\right)$. We join these two $\left\{n_{0}\right\}$-cubes by adding the relations $F_{n_{1}}\left(a_{0}, a_{1}\right)$ and $F_{n_{1}}\left(b_{0}, b_{1}\right)$ and no other relations (except those forced by symmetry). Notice that the function $f_{n_{1}}$ induced by $F_{n_{1}}$ and defined by $f_{n_{1}}\left(a_{i}\right)=$ $a_{1-i}$ and $f_{n_{1}}\left(b_{i}\right)=b_{1-i}$ for $i \leq 1$ is both an $\mathcal{L}$-isomorphism between the two $\left\{n_{0}\right\}$ cubes and an $\mathcal{L}$-automorphism of the $S$-cube. Similarly, the function $f_{n_{0}}$ induced by $F_{n_{0}}$ is an $\mathcal{L}$-automorphism of the $S$-cube. Combining these automorphisms, it is clear that any two elements of the $S$-cube are automorphic. (We could get an isomorphic $S$-cube by starting with two $\left\{n_{1}\right\}$-cubes and connecting them using $F_{n_{0}}$. Therefore, the roles played by $n_{0}$ and $n_{1}$ are actually symmetric.)

For one last example, suppose $S=\left\{n_{0}, n_{1}, n_{2}\right\}$. To construct the $S$-cube, we begin with two $\left\{n_{0}, n_{1}\right\}$-cubes given by $a_{0}, a_{1}, b_{0}$ and $b_{1}$ (with relations as above) and by $a_{0}^{\prime}, a_{1}^{\prime}, b_{0}^{\prime}$ and $b_{1}^{\prime}$ (with relations defined similarly). We join these two cubes by adding the relations $F_{n_{2}}\left(a_{i}, a_{i}^{\prime}\right)$ and $F_{n_{2}}\left(b_{i}, b_{i}^{\prime}\right)$ for $i \leq 1$ and no other relations (except those forced by symmetry). As above, the function $f_{n_{2}}$ induced by $F_{n_{2}}$ is both an $\mathcal{L}$-isomorphism between the $\left\{n_{0}, n_{1}\right\}$-cubes and an $\mathcal{L}$-automorphism of the $S$-cube. Similarly, the functions $f_{n_{0}}$ and $f_{n_{1}}$ induced by $F_{n_{0}}$ and $F_{n_{1}}$ are $\mathcal{L}$ automorphisms of the $S$-cube. Combining these automorphisms, it is clear that any two elements of the $S$-cube are automorphic.

We now give the formal definition of an $S$-cube.

Definition 3. Given a nonempty (finite or infinite) subset $S \subseteq \omega$, we let $S=$ $\left\{n_{0}<n_{1}<\ldots\right\}$ be a (not necessarily effective) enumeration of $S$ in order, and set $S_{k}=\left\{n_{0}, \ldots, n_{k}\right\}$ (for $k<|S|$ if $S$ is finite). We define the notion of an $S$-cube by induction on $k$.

An $S_{0}$-cube is a 2-element subset $\{x, y\}$ of an $\mathcal{L}$-model such that $F_{n_{0}}(x, y)$ (coding $f_{n_{0}}(x)=y$ and $\left.f_{n_{0}}(y)=x\right)$ and $\neg F_{n}(x, y)$ for all $n \neq n_{0}$.

An $S_{k+1}$-cube is the disjoint union of two $S_{k}$-cubes $C_{0}$ and $C_{1}$ such that $F_{n_{k+1}}$ induces an $\mathcal{L}$-isomorphism between $C_{0}$ and $C_{1}$, and such that $\neg F_{n}\left(x_{0}, x_{1}\right)$ for all $n \neq n_{k+1}$, all $x_{0} \in C_{0}$ and all $x_{1} \in C_{1}$.

For infinite $S$, we define an $S$-cube to be the "limit" of the $S_{k}$-cubes, i.e., an infinite subset $A$ of an $\mathcal{L}$-model such that for all $k \in \omega$, every element $x \in A$ is an element of a unique $S_{k}$-cube $\subset A$ and such that any two elements $x_{0}, x_{1} \in A$ are connected by a finite chain of $F_{i}$-relations.

Notice that if $X \subsetneq Y$, then any $Y$-cube contains $X$-cubes. We will use the term $X$-component to refer to an $X$-cube which is not contained in a larger cube. (Intuitively, an $X$-component is a connected component of the model viewed as a graph.) There are two important properties of an $S$-component. First, any two elements of an $X$-component are automorphic. Second, if $x$ is an element of an $S$-component, then there exists a $y$ such that $F_{n}(x, y)$ if and only if $n \in S$. 
For the rest of this paper, $\mathcal{F}$ denotes an a.e. family of finite subsets of $\omega$ with an infinite limit $I_{\mathcal{F}}$. We code such a family into an $\mathcal{L}$-theory as follows. Let $\mathcal{A}_{0, \mathcal{F}}$ denote the $\mathcal{L}$-structure consisting of a single $X$-component for each $X \in \mathcal{F}$ and let $T_{\mathcal{F}}$ be the theory of this structure. We first examine some model theoretic properties of such a theory.

Lemma 4. $\mathcal{A}_{0, \mathcal{F}}$ is the atomic model of $T_{\mathcal{F}}$. Furthermore, for any nonatomic model $\mathcal{M} \models T_{\mathcal{F}}$ and any $m \in \mathcal{M} \backslash \mathcal{A}_{0, \mathcal{F}}, m$ sits inside an $I_{\mathcal{F}}$-component. (Thus any model of $T_{\mathcal{F}}$ can be denoted as $\mathcal{A}_{\kappa, \mathcal{F}}$ and is the disjoint union of the atomic

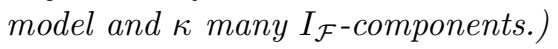

Proof. To show that $\mathcal{A}_{0, \mathcal{F}}$ is the atomic model of $T_{\mathcal{F}}$, fix $a \in \mathcal{A}_{0, \mathcal{F}}$ and $X \in \mathcal{F}$ such that $a$ is an element of the $X$-component in $A_{0, \mathcal{F}}$. Since $I_{\mathcal{F}}$ is infinite, we can fix $i \in I_{\mathcal{F}} \backslash X$. Since $\mathcal{F}$ is an a.e. family, there are finitely many $Y \in \mathcal{F}$ such that $i \notin Y$, and we let $X_{0}, X_{1}, \ldots, X_{k-1}$ list these elements of $\mathcal{F}$ which are not equal to $X$. For each $0 \leq j<k$, fix $n_{j}$ such that either $n_{j} \in X \backslash X_{j}$ or $n_{j} \in X_{j} \backslash X$. Let $\psi_{a}(x)$ be the formula that says $\exists y\left(F_{m}(x, y)\right)$ for each $m \in X, \neg \exists y\left(F_{i}(x, y)\right)$ for our fixed element $i \in I_{\mathcal{F}} \backslash X$, and $\neg \exists y\left(F_{n_{j}}(x, y)\right)$ for each $0 \leq j<k$ such that the fixed element $n_{j}$ satisfies $n_{j} \in X_{j} \backslash X$.

Clearly, $a$ satisfies $\psi_{a}(x)$. Moreover, if $b \in A_{0, \mathcal{F}}$ and $b$ is not in the $X$-component, then we claim that $b$ does not satisfy $\psi_{a}(x)$. Fix $Y \in \mathcal{F}$ such that $b$ is in the $Y$ component of $\mathcal{A}_{0, \mathcal{F}}$. If $i \in Y$, then $b$ fails to satisfy $\neg \exists y\left(F_{i}(x, y)\right)$. Suppose $Y=X_{j}$ for some $0 \leq j<k$. If $n_{j} \in X \backslash X_{j}$, then $b$ fails to satisfy the $\exists y\left(F_{n_{j}}(x, y)\right)$ conjunct in $\psi_{a}(x)$. If $n_{j} \in X_{j} \backslash X$, then $b$ fails to satisfy the $\neg \exists y\left(F_{n_{j}}(x, y)\right)$ conjunct in $\psi_{a}(x)$. Therefore, the only elements of $\mathcal{A}_{0, \mathcal{F}}$ which satisfy $\psi_{a}(x)$ are the elements of the $X$-component.

Thus, for each $k \notin X, T_{\mathcal{F}}$ contains the sentence $\neg \exists x, y\left(\psi_{a}(x) \wedge F_{k}(x, y)\right)$. Hence, if $\mathcal{M}$ is any model of $T_{\mathcal{F}}$ and $b \in \mathcal{M}$ satisfies $\psi_{a}(x)$, then $b$ is an element of an $X$-component. Since $\mathcal{M}$ must contain such an element $b$, we have that $\mathcal{M}$ contains an $X$-component. It follows that $A_{0, \mathcal{F}}$ embeds into $\mathcal{M}$, so $\mathcal{A}_{0, \mathcal{F}}$ is the prime (and hence atomic) model of $T_{\mathcal{F}}$. Furthermore, because $T_{\mathcal{F}}$ says that there are exactly $2^{|X|}$ many elements which satisfy $\psi_{a}(x)$, the principal type satisfied by an element of an $X$-component is algebraic over $\emptyset$.

Next, we show that if $\mathcal{M} \models T_{\mathcal{F}}$ is a nonprime model and $m \in \mathcal{M} \backslash \mathcal{A}_{0, \mathcal{F}}$, then $m$ is contained in an $I_{\mathcal{F}}$-component. Let $I_{\mathcal{F}}=\left\{i_{0}<i_{1}<i_{2}<\cdots\right\}$ be a (not necessarily effective) enumeration of $I_{\mathcal{F}}$. For each $k \in \omega$, we let $C_{k}$ denote the $Y$-cube where $Y=\left\{i_{0}, i_{1}, \ldots, i_{k}\right\}$.

We think of $\mathcal{M}$ containing an edge between any elements $a$ and $b$ if $F_{i}(a, b)$ holds for some $i$ and we use graph-theoretic language. We define a modified notion of "open balls" in $\mathcal{M}$. For any $a \in \mathcal{M}$ and $k \in \omega, B(a, k)$ is the set of all $b \in \mathcal{M}$ such that the distance between $a$ and $b$ as measured by the relations $F_{0}, \ldots, F_{i_{k}}$ is $\leq k$.

By the properties of $\mathcal{F}$, for any fixed $k \in \omega$, almost all $a \in \mathcal{M}$ satisfy $B(a, k) \cong$ $C_{k}$. For each $k \in \omega$, the same finite number of exceptional points $a$ for which $B(a, k) ¥ C_{k}$ exist in $\mathcal{M}$ as in $\mathcal{A}_{0, \mathcal{F}}$ and hence these exceptional points all come from the finite components of $\mathcal{A}_{0, \mathcal{F}}$ sitting inside $\mathcal{M}$. Since any point $a \in \mathcal{M} \backslash \mathcal{A}_{0, \mathcal{F}}$ satisfies $B(a, k) \cong C_{k}$ for all $k \in \omega$, each such $a$ sits inside an $I_{\mathcal{F}}$-component. Furthermore, since any two points in an $I_{\mathcal{F}}$-component are automorphic, any two elements in $\mathcal{M} \backslash \mathcal{A}_{0, \mathcal{F}}$ satisfy the same 1-type. Thus, there is a unique nonprincipal (and nonalgebraic) 1-type in $\mathcal{M}$ for any nonprime model $\mathcal{M}$ of $T_{\mathcal{F}}$. 
Lemma 5. $T_{\mathcal{F}}$ is trivial and strongly minimal.

Proof. To see that $T_{\mathcal{F}}$ is strongly minimal, let $\psi(x)$ be any formula, possibly with parameters $\bar{b}$ from a model $\mathcal{M}=T_{\mathcal{F}}$. We need to show that either $\left\{m \in \mathcal{M}^{\prime} \mid\right.$ $\left.\mathcal{M}^{\prime} \models \psi(m)\right\}$ or $\left\{m \in \mathcal{M}^{\prime} \mid \mathcal{M}^{\prime} \models \neg \psi(m)\right\}$ is finite for any elementary extension $\mathcal{M}^{\prime}$ of $\mathcal{M}$. Let $\mathcal{L}_{\bar{b}}$ be the restriction of our language to those edge relations occurring in $\psi(x)$. Then the reduct $\mathcal{M}^{\prime} \uparrow \mathcal{L}_{\bar{b}}$ is the disjoint union of (maximal) $X$-cubes for various finite sets $X$ containing only indices of edge relations from the finite set of indices $I_{0}$ of edge relations mentioned in $\psi(x)$, and all but finitely many of these cubes are $X_{0}$-cubes for $X_{0}=I_{\mathcal{F}} \cap I_{0}$ and do not contain a parameter from $\bar{b}$. Since the stabilizer of $\bar{b}$ in the automorphism group of $\mathcal{M}^{\prime}\left\lceil\mathcal{L}_{\bar{b}}\right.$ is 1-transitive on the union $U_{0}$ of all those $X_{0}$-cubes, $U_{0}$ is a cofinite subset of $\mathcal{M}^{\prime}$ which is completely contained in either $\left\{m \in \mathcal{M}^{\prime} \mid \mathcal{M}^{\prime} \models \psi(m)\right\}$ or $\left\{m \in \mathcal{M}^{\prime} \mid \mathcal{M}^{\prime} \models \neg \psi(m)\right\}$, establishing our claim of strong minimality.

It remains to show that $T_{\mathcal{F}}$ is trivial, or in other words, that $\operatorname{acl}(A)=\bigcup_{a \in A} \operatorname{acl}(a)$ for all $A \subseteq \mathcal{M}$ with $\mathcal{M} \models T_{\mathcal{F}}$. We have already pointed out that acl $(\emptyset)$ is equal to the set of all finite $X$-components in $\mathcal{M}$. (That is, $\operatorname{acl}(\emptyset)=\mathcal{A}_{0, \mathcal{F}} \prec \mathcal{M}$.) We claim that $\operatorname{acl}(A)$ is equal to the set of all finite $X$-components in $\mathcal{M}$ together with the set of all $I_{\mathcal{F}}$-components in $\mathcal{M}$ which intersect $A$. The fact that $T_{\mathcal{F}}$ is trivial follows immediately from this claim.

To see the claim, notice that if $b \in \mathcal{M}$ is in the same $I_{\mathcal{F}}$-component as $a \in A$, then there is a path in $\mathcal{M}$ from $a$ to $b$ (using various relations $F_{i}$ ) and that $b$ is the unique point at the end of such a path. Therefore, the set of all $I_{\mathcal{F}}$-components which intersect $A$ are contained in $\operatorname{acl}(A)$. Furthermore, if $b$ is in an $I_{\mathcal{F}}$-component which does not intersect $A$, then for any other $c$ in the same $I_{\mathcal{F}}$-component as $b$, there is an automorphism of $\mathcal{M}$ which sends $b$ to $c$ and fixes the finite $X$-components and the $I_{\mathcal{F}}$-components which intersect $A$. Therefore, $b$ and $c$ realize the same type over $A$; this type is not algebraic over $A$ and neither $b$ nor $c$ is in $\operatorname{acl}(A)$.

We next examine some computability-theoretic properties of models of $T_{\mathcal{F}}$.

Definition 6. A c.e. enumeration of a countable family $\mathcal{G}$ of subsets of $\omega$ is a uniformly c.e. sequence of sets $X_{i}$ such that $\mathcal{G}=\left\{X_{i} \mid i \in \omega\right\}$. If $i \neq j$ implies $X_{i} \neq X_{j}$, then we call this enumeration a c.e. Friedberg enumeration of $\mathcal{G}$. (We note that in Russian terminology, a c.e. enumeration is frequently called a computable numbering.)

Lemma 7. If there is a c.e. Friedberg enumeration of $\mathcal{F}$, then $T_{\mathcal{F}}$ has a computable prime model.

Proof. Given a c.e. Friedberg enumeration of $\mathcal{F}$, we construct a computable prime model of $T_{\mathcal{F}}$ by building the $X$-component for each $X \in \mathcal{F}$ as it is enumerated. That is, we do not need to specify all of the $X$-component at once, but can build it in stages as the (finitely many) elements of $X$ appear.

The following lemma gives the key property for verifying that theories $T_{\mathcal{F}}$ for appropriate families $\mathcal{F}$ can code additional information into their nonprime models.

Lemma 8. If $\mathcal{A}$ is a nonprime model of $T_{\mathcal{F}}$, then $I_{\mathcal{F}}$ is c.e. relative to the atomic diagram of $\mathcal{A}$.

Proof. Fix an element $c$ in one of the $I_{\mathcal{F}}$-components in $\mathcal{A}$. Using the atomic diagram of $\mathcal{A}$, we enumerate the numbers $n \in \omega$ such that there exists an element 
$x$ in $\mathcal{A}$ which is related to $c$ by $F_{n}$. By the definition of the $I_{\mathcal{F}}$-component, this procedure enumerates $I_{\mathcal{F}}$.

By Lemma 8 , we can code $0^{\prime \prime}$ into the nonprime models of $\mathcal{F}$ by making $0^{\prime \prime}$ computable from any enumeration of $I_{\mathcal{F}}$. We do this by making $I_{\mathcal{F}}$ code the graph of a function which grows fast enough to compute $0^{\prime \prime}$.

We develop a suitable computable approximation to $0^{\prime \prime}$. Fix the canonical uniformly c.e. sequence of all c.e. sets $W_{n}$ for $n \in \omega$ so that $\left\{n \mid W_{n}\right.$ is finite $\} \equiv_{T} 0^{\prime \prime}$. We let $W_{n, s}$ denote the elements of $W_{n}$ that have been enumerated by stage $s$. Let $f(n)$ be the least $s$ such that the finite sets among $W_{0}, \ldots, W_{n}$ have been completely enumerated by stage $s$.

Lemma 9. For any function $g$ which dominates $f, 0^{\prime \prime} \leq_{T} g \oplus 0^{\prime}$.

Proof. Let $k \in \omega$ be such that $f(x) \leq g(x)$ for all $x \geq k$. To determine whether $n \in 0^{\prime \prime}$ for $n \geq k$, ask $0^{\prime}$ whether $W_{n}$ gets an element after stage $g(n)$. The answer to this question is no if and only if $n \in 0^{\prime \prime}$.

Lemma 10. There is a computable function $f(n, s)$ which satisfies the following properties:

(1) $f(n)=\liminf _{s} f(n, s)$.

(2) For every $k>f(n)$, there is a stage $s_{k}$ such that for all $t \geq s_{k}$ either $f(n, t)=f(n)$ or $f(n, t)>k$.

Proof. The following procedure to define $f(n, s)$ is uniform in $n$ and takes place in stages $s$. Fix $n$, let $I_{j}, j<2^{n+1}$, list the subsets of $[0, n]$ and let $I^{*}=\{j \leq n \mid$ $\left.\left|W_{j}\right|=\infty\right\}$. We think of each $I_{j}$ as a potential guess at $I^{*}$.

At stage $s$, consider each $I_{j}$. If a new element is enumerated into $W_{i}$ at stage $s$ and $i \in I_{j}$, then we declare $i \in I_{j}$ to be checked in $I_{j}$. (Once $i$ is checked in $I_{j}$, it remains checked in $I_{j}$ at future stages until $I_{j}$ acts and we explicitly declare $i$ to be unchecked in $I_{j}$.) If all of the elements of $I_{j}$ are checked in $I_{j}$, then we declare $I_{j}$ to be ready. Notice that the empty set is ready at every stage.

Let $j$ denote the index such that $I_{j}$ is ready and $I_{j}$ has acted least recently. (This index is unique unless there is more than one $j$ such that $I_{j}$ is ready and $I_{j}$ has never acted. In that case, let $I_{j}$ be the least such index.) We let $I_{j}$ act at stage $s$ as follows. Define

$$
\begin{gathered}
G(n, s)=\max \left\{t<s \mid W_{n, t} \neq W_{n, t+1}\right\}, \\
f(n, s)=\max \left\{G(k, s) \mid k \leq n \wedge k \notin I_{j}\right\} .
\end{gathered}
$$

Declare $I_{j}$ unready and uncheck each $i \in I_{j}$. (If $i \in I_{k}$ is checked in $I_{k}$ for $k \neq j$, then $i$ remains checked in $I_{k}$.)

This completes the description of $f(n, s)$. It remains to verify properties (1) and (2). Let $S^{*}=\left\{s \mid I_{j}=I^{*}\right.$ acts at $\left.s\right\}$. By the construction, $S^{*}$ is infinite and

$$
\lim _{s \in S^{*}} f(n, s)=f(n) .
$$

To verify $\liminf s_{s} f(n, s)=\lim _{s \in S^{*}} f(n, s)$ and property (2), we need to consider what happens when each $I_{j} \neq I^{*}$ acts. We split this analysis into two cases: when $\exists i\left(i \in I_{j}-I^{*}\right)$ and when $I_{j} \subseteq I^{*}$ but $\exists i\left(i \in I^{*}-I_{j}\right)$.

First, suppose $\exists i\left(i \in I_{j}-I^{*}\right)$. Since $i \notin I^{*}, W_{i}$ is finite and hence $i \in I_{j}$ is only checked finitely often in $I_{j}$. Therefore, $I_{j}$ only acts finitely often and has no effect on $f(n, s)$ for large enough values of $s$. 
Second, suppose $I_{j} \subseteq I^{*}$ and $\exists i\left(i \in I^{*}-I_{j}\right)$. Let $S_{j}=\left\{s \mid I_{j}\right.$ acts at $\left.s\right\}$. Since $I_{j} \subseteq I^{*}, S_{j}$ is infinite. Since $i \in I^{*}, W_{i}$ is infinite and $\lim _{s} G(i, s)=\infty$. Since $i \notin I_{j}$, at each $s \in S_{j}, f(n, s) \geq G(i, s)$. Therefore, $\lim _{s \in S_{j}} f(n, s)=\infty$.

Properties (1) and (2) follow easily from these observations.

We need to add one further piece to our approximation of $0^{\prime \prime}$. (By appealing to the s-m-n theorem, we could have skipped this step, but it is perhaps easier to continue the theme of dominating functions.) We let $h(n)$ be the least stage $s$ for which $K_{s}\left\lceil n+1=K\left\lceil n+1\right.\right.$. (Here, $K=\left\{e \mid \varphi_{e}(e)\right.$ halts $\}$ denotes the usual halting set for the partial computable functions.) Since $h(n)$ is a $\Delta_{2}^{0}$-function, it has a computable approximation $h(n, s)$ such that $\lim _{s} h(n, s)=h(n)$. Finally, let $a(n, s)$ be the computable function defined by $a(n, s)=\max \{f(n, s), h(n, s)\}$.

Lemma 11. The computable function a $(n, s)$ satisfies the following properties:

(1) $a(n)=\liminf _{s} a(n, s)$ exists and for all $n, a(n) \geq f(n), h(n)$.

(2) For every $k>a(n)$, there is a stage $s_{k}$ such that for all $t \geq s_{k}$, either $a(n, t)=a(n)$ or $a(n, t)>k$.

(3) For any function b( $n)$ dominating $a(n), 0^{\prime \prime} \leq_{T} b$. In particular, $0^{\prime \prime} \leq_{T} a$.

Proof. Properties (1) and (2) follow from Lemma 10 and the fact that

$$
h(n)=\lim _{s} h(n, s) .
$$

Property (3) follows from the fact that if $b(n)$ dominates $a(n)$, then $b(n)$ dominates both $h(n)$ and $f(n)$. The fact that $b(n)$ dominates $h(n)$ gives $0^{\prime} \leq_{T} b$. Combining this fact with Lemma 9 gives $0^{\prime \prime} \leq_{T} b$.

We use the function $a(n, s)$ to prove Theorem 1 We start by fixing a particular family $\mathcal{F}$.

Definition 12. From now on, we fix the family $\mathcal{F}$ to be the set $\left\{X_{s} \mid s \in \omega\right\}$ where $X_{s}$ is the set of all pairs $\langle n, m\rangle$ such that $n \leq s$ and

$$
\exists t \geq s[a(n, t)=m \wedge \forall u(s \leq u \leq t \rightarrow a(n, t) \leq a(n, u))] .
$$

Let $I_{\mathcal{F}}=\{\langle n, m\rangle \mid a(n)=m\}$.

Lemma 13. $\mathcal{F}$ is an a.e. family of finite subsets of $\omega$ with infinite limit $I_{\mathcal{F}}$.

Proof. Each $X_{s}$ is finite because $\langle n, m\rangle \in X_{s}$ implies that $n \leq s$ and $m \leq a(n, s)$. Therefore, $\mathcal{F}$ is a family of finite sets. Furthermore, $I_{\mathcal{F}}$ is clearly infinite. It remains to show that $\mathcal{F}$ is an a.e. family with limit $I_{\mathcal{F}}$.

On the one hand, assume that $\langle n, m\rangle \in I_{\mathcal{F}}$. We show that $\langle n, m\rangle \in X_{s}$ for almost all $s$. By the definition of $I_{\mathcal{F}}, a(n)=m$. By Lemma 11, we can fix $s_{0}$ such that for all $s \geq s_{0}, a(n, s) \geq a(n)$. For every $s \geq s_{0}$, there is a $t \geq s$ such that $a(n, t)=a(n)$ and hence the pair $\langle n, a(n)\rangle$ is eventually enumerated into $X_{s}$ for each $s \geq s_{0}$.

On the other hand, assume that $\langle n, m\rangle \notin I_{\mathcal{F}}$. We show that $\langle n, m\rangle \in X_{s}$ for only finitely many $s$. By the definition of $I_{\mathcal{F}}, a(n) \neq m$. Since $a(n)=\liminf _{s} a(n, s)$, this claim is clear in the case when $m<a(n)$. If $a(n)<m$, then by Lemma 11, there is a stage $s_{m}$ such that for all $s \geq s_{m}$, either $a(n, s)=a(n)$ or $a(n, s)>m$. Therefore, for all $s \geq s_{m}, a(n, s) \neq m$. It follows that $\langle n, m\rangle \notin X_{s}$ for any $s \geq s_{m}$.

By Lemma 5 , the family $\mathcal{F}$ generates a theory $T_{\mathcal{F}}$ which is trivial and strongly minimal. 
Lemma 14. There is a c.e. Friedberg enumeration of $\mathcal{F}$.

Proof. This lemma follows because $a(n, s)$ is a computable function and hence the definition of $X_{s}$ is uniformly $\Sigma_{1}^{0}$.

By Lemma 7 , $T_{\mathcal{F}}$ has a computable prime model.

Lemma 15. If $\mathcal{A}$ is a nonprime model of $T_{\mathcal{F}}$, then $0^{\prime \prime}$ is computable from the atomic diagram of $\mathcal{A}$.

Proof. By Lemma 8 , we can enumerate $I_{\mathcal{F}}$ from the atomic diagram of $\mathcal{A}$ and hence we can enumerate the graph of $a(n)$ from the atomic diagram of $\mathcal{A}$. Therefore, we can compute the function $a(n)$ from the diagram of $\mathcal{A}$ and thus can compute $0^{\prime \prime}$ by Lemma 11.

This completes the proof of Theorem 1

\section{Some REMARKS ON AXIOMATIZABILITY}

We now conclude with some remarks on axiomatizability by formulas of various quantifier-complexity.

Definition 16. An $\mathcal{L}$-theory $T$ has a quantifier-depth 1 axiomatization if $T$ is equivalent to a set $S$ of sentences, each of which is a Boolean combination of universal sentences.

Definition 17. An $\mathcal{L}$-theory $T$ is Skolemized if for every $\mathcal{L}$-formula $\varphi(x, \bar{y})$ there are finitely many terms $\tau_{1}(\bar{y}), \ldots, \tau_{n}(\bar{y})$ such that

$$
T \models \forall \bar{y}\left[\exists x \varphi(x, \bar{y}) \rightarrow \bigvee_{i} \varphi\left(\tau_{i}(\bar{y}), \bar{y}\right)\right] .
$$

The Tarski-Vaught criterion for being an elementary substructure, coupled with an easy compactness argument, entails that $T$ is Skolemized if and only if every substructure of every model of $T$ is an elementary substructure. Recalling that a theory $T$ has a universal axiomatization if and only if the class of models of $T$ is closed under substructures, it follows immediately that any Skolemized theory has a universal axiomatization, and the converse holds whenever the theory is model complete.

Lemma 18. The following are equivalent for any structure $\mathcal{M}$ such that $\operatorname{Th}\left(\mathcal{M}_{M}\right)$ is model complete:

(1) $\operatorname{Th}\left(\mathcal{M}_{M}\right)$ has a quantifier-depth 1 axiomatization;

(2) $\operatorname{Th}\left(\mathcal{M}_{M}\right)$ has a universal axiomatization;

(3) $\operatorname{Th}\left(\mathcal{M}_{M}\right)$ is Skolemized.

Proof. (2) $\Rightarrow(1)$ is trivial and (3) $\Leftrightarrow(2)$ follows from our comments above. Finally, (1) $\Rightarrow(2)$ holds for any structure $\mathcal{M}$. Indeed, suppose (1) holds for $\mathcal{M}$ and choose $\mathcal{M}^{\prime} \models \operatorname{Th}\left(\mathcal{M}_{M}\right)$ and any $\mathcal{L}_{M}$-substructure $\mathcal{N}$ of $\mathcal{M}^{\prime}$. We may assume that $M \subseteq$ $N \subseteq M^{\prime}$ and $\mathcal{M} \preceq \mathcal{M}^{\prime}$. It suffices to show that $\mathcal{N} \models \operatorname{Th}\left(\mathcal{M}_{M}\right)$. Let $\sigma$ be any universal $\mathcal{L}_{M}$-sentence. Clearly, if $\mathcal{N} \models \sigma$, then $\mathcal{M} \models \sigma$ as well. Conversely, if $\mathcal{M} \models \sigma$, then $\mathcal{M}^{\prime} \models \sigma$ by elementarity, hence $\mathcal{N} \models \sigma$ as well. Thus, $\mathcal{M}$ and $\mathcal{N}$ agree on all universal $\mathcal{L}_{M}$-sentences, hence on all Boolean combinations of universal $\mathcal{L}_{M}$-sentences, so $\mathcal{N} \models \operatorname{Th}\left(\mathcal{M}_{M}\right)$ by $(1)$. 
By adding dummy variables as needed, it is easily verified that for any $\mathcal{L}$ structure $\mathcal{M}, \operatorname{Th}\left(\mathcal{M}_{M}\right)$ is Skolemized if and only if for every $\mathcal{L}$-formula $\varphi(x, \bar{y})$ there are finitely many $\mathcal{L}$-terms $\tau_{1}\left(\bar{y}, \bar{z}_{1}\right), \ldots, \tau_{n}\left(\bar{y}, \bar{z}_{n}\right)$ such that

$$
\operatorname{Th}(\mathcal{M}) \models \exists \bar{z}_{1} \ldots \exists \bar{z}_{n} \forall \bar{y}\left[\exists x \varphi(x, \bar{y}) \rightarrow \bigvee_{i} \varphi\left(\tau_{i}\left(\bar{y}, \bar{z}_{i}\right), \bar{y}\right)\right] .
$$

Thus, the property of $\operatorname{Th}\left(\mathcal{M}_{M}\right)$ being Skolemized is preserved under elementary equivalence.

Proposition 19. The following are equivalent for any trivial, strongly minimal (complete) $\mathcal{L}$-theory $T$ :

(1) $\operatorname{Th}\left(\mathcal{M}_{M}\right)$ has a quantifier-depth 1 axiomatization for some $\mathcal{M} \models T$.

(2) $\operatorname{Th}\left(\mathcal{M}_{M}\right)$ has a quantifier-depth 1 axiomatization for all $\mathcal{M} \models T$.

(3) For all $M \subseteq A \subseteq M^{\prime}$ such that $\mathcal{M} \preceq \mathcal{M}^{\prime}$ are models of $T$, and for every $b \in \operatorname{acl}_{\mathcal{M}^{\prime}}(A)$, there are an $\mathcal{L}$-term $\tau(\bar{y})$ and $\bar{a} \in A$ with $\mathcal{M}^{\prime} \models \tau(\bar{a})=b$.

Proof. Recall from Goncharov/Harizanov/Laskowski/Lempp/McCoy GHLLM03. that $\operatorname{Th}\left(\mathcal{M}_{M}\right)$ is model complete for every model $\mathcal{M}$ of $T$. Combining this with the preservation of being Skolemized under elementary equivalence, $(1) \Leftrightarrow(2)$ follows from the lemma.

Now assume that (2) holds. Choose $\mathcal{M}, \mathcal{M}^{\prime}, A, b$ as in (3) and let $\mathcal{N}$ be the smallest substructure of $\mathcal{M}^{\prime}$ containing $A \cup\{b\}$. Since $\operatorname{Th}\left(\mathcal{M}_{M}\right)$ is model complete, Lemma 18 implies that $\operatorname{Th}\left(\mathcal{M}_{M}\right)$ is Skolemized, hence $\mathcal{N} \preceq \mathcal{M}^{\prime}$. Thus acl $\mathcal{M}^{\prime}(A)=$ $\operatorname{acl}_{\mathcal{N}}(A)=N$, so (3) is satisfied.

Finally, suppose (3) holds and fix $\mathcal{M} \models T$. We show that $\operatorname{Th}\left(\mathcal{M}_{M}\right)$ has a universal axiomatization by choosing $\mathcal{M}^{\prime} \succeq \mathcal{M}$ and $N$ such that $M \subseteq N \subseteq M^{\prime}$ as $\mathcal{L}$-structures. By (3), $N$ is infinite and algebraically closed in the $\mathcal{L}_{M}$-structure $\mathcal{M}_{M}^{\prime}$, which has a strongly minimal theory. Thus $\mathcal{N} \models \operatorname{Th}\left(\mathcal{M}_{M}\right)$ as required.

\section{ACKNOWLEDGMENTS}

The authors thank the referee for helpful comments and for simplifying the proof of Lemma 10.

\section{REFERENCES}

[BL71] Baldwin, J.T. and Lachlan, A.H., On strongly minimal sets, J. Symbolic Logic, 36 (1971), 79-96. MR0286642 (44:3851)

[Bu96] Buechler, Steven A., Essential Stability Theory, Springer-Verlag, Heidelberg, 1996. MR:1416106 (98j:03050)

[CK90] Chang, Chen Chung and Keisler, H. Jerome, Model theory. Third edition, Studies in Logic and the Foundations of Mathematics, North-Holland, Amsterdam, 1990. MR.1059055 (91c:03026)

[Go78] Goncharov, Sergey S., Constructive models of $\aleph_{1}$-categorical theories, Mat. Zametki 23 (1978), 885-888. MR.502056 (80g:03029)

[GK04] Goncharov, Sergey S. and Khoussainov, Bakhadyr M., Complexity of categorical theories with computable models, Algebra and Logic, 43 (2004), 650-665, 758-759. MR.2135386 (2005m:03066)

[GHLLM03] Goncharov, Sergey S.; Harizanov, Valentina S.; Laskowski, Michael C.; Lempp, Steffen; and McCoy, Charles F. D., Trivial, strongly minimal theories are model complete after naming constants, Proc. Amer. Math. Soc. 131 (2003), 3901-3912. MR1999939 (2004g:03054)

[Ha74] Harrington, Leo, Recursively presented prime models, J. Symbolic Logic 39 (1974), 305-309. MR0351804(50:4292) 
[HLZ99] Herwig, Bernhard; Lempp, Steffen; and Ziegler, Martin, Constructive models of uncountably categorical theories, Proc. Amer. Math. Soc. 127 (1999), 3711-3719. MR.1610909 (2000b:03129)

[Kh74] Khisamiev, Nazif G., On strongly constructive models of decidable theories, Izv. Akad. Nauk Kazakh. SSR Ser. Fiz.-Mat. 35 (1) (1974), 83-84.

[KNS97] Khoussainov, Bakhadyr M.; Nies, André O.; and Shore, Richard A., Computable models of theories with few models, Notre Dame J. Formal Logic 38 (1997), 165178. MR:1489408 (99c:03049)

[Kuta] Kueker, David W., Weak invariance and model completeness relative to parameters, in preparation.

[Ku80] Kudaibergenov, Kanat Zh., Constructivizable models of undecidable theories, Sibirsk. Mat. Zh. 21 (5) (1980), 155-158, 192. MR592228 (82h:03040)

[Ma89] Marker, David, Non $\Sigma_{n}$ axiomatizable almost strongly minimal theories, J. Symbolic Logic 54 (1989), 921-927. MR1011179 (90g:03037)

[Mo65] Morley, Michael, Categoricity in power, Trans. Amer. Math. Soc. 114 (1965), 514538. MR.0175782 (31:58)

[Ni99] Nies, André, A new spectrum of recursive models, Notre Dame J. Formal Logic 40 (1999), 307-314. MR1845630(2002e:03066)

Department of Computer Science, University of Auckland, Auckland, New Zealand

E-mail address: bmk@cs.auckland.ac.nz

Department of Mathematics, University of Maryland, College Park, Maryland 20742

E-mail address: mcl@math.umd.edu

Department of Mathematics, University of Wisconsin, Madison, Wisconsin 53706

E-mail address: lempp@math.wisc.edu

Department of Mathematics, University of Connecticut, Storrs, Connecticut 06269

E-mail address: solomon@math.uconn.edu 